\title{
O TOMATE COMO ALIMENTO - CADEIA PRODUTIVA E RESÍDUOS DE AGROTÓXICOS
}

GRAZIELA CRISTINA ROSSI MOURA-ANDRADE*

MARÍLIA OETTERER**

VALDEMAR LUIZTORNISIELO***

\begin{abstract}
O objetivo deste trabalho foi efetuar revisão de literatura sobre a importância do tomate e seus derivados na dieta alimentar, mostrar a influência da cadeia agroindustrial do tomate no âmbito do agronegócio brasileiro e apontar método eficiente para detecção de resíduos de agrotóxicos nessa matriz. Para seu consumo seguro são necessários, além da aplicação das boas práticas agrícolas no seu cultivo, informações sobre o monitoramento de resíduos de agrotóxicos que garantam a qualidade do alimento submetido a tratamentos com agrotóxicos. A disponibilização de métodos multirresíduos que promovam a detecção de várias classes de agrotóxicos em matrizes complexas, como o QuEChERS, oferecem várias vantagens na determinação de resíduos de agrotóxicos em alimentos, podendo ser usado como método de rotina em diversos laboratórios.
\end{abstract}

PALAVRAS-CHAVE: TOMATE; LICOPENO; RESÍDUOS DE AGROTÓXICOS; ANÁLISES MULTIRRESÍDUOS.

* Doutoranda em Ciências, Departamento de Ecotoxicologia, Centro de Energia Nuclear na Agricultura, USP, Piracicaba, SP (e-mail: grazielacrm@yahoo.com.br).

** Doutora em Ciências dos Alimentos, Professora Titular, Departamento de Agroindústria, Alimentos e Nutrição, Escola Superior de Agricultura Luiz de Queiroz, USP, Piracicaba, SP (e-mail: moettere@esalq.usp.br).

*** Doutor em Tecnologia Nuclear, Professor Associado, Centro de Energia Nuclear na Agricultura, USP, Piracicaba, SP (e-mail: vltornis@cena.usp.br). 


\section{INTRODUÇÃO}

A cadeia agroindustrial do tomate está entre as mais importantes no âmbito do agronegócio brasileiro. A cultura do tomate para processamento industrial movimenta as indústrias paralelas de insumos, embalagens, máquinas agrícolas e equipamentos de irrigação. Como matéria-prima, o tomate para processamento de derivados representa a principal atividade geradora de renda para grande número de produtores, tornando-se significativa fonte de renda regional (MELO e VILELA, 2004)

O Brasil é o principal produtor de tomate da América do Sul, seguido pelo Chile e pela Argentina. No início da década de 90 , a área plantada de tomate para processamento industrial foi de 27 mil hectares. Desse total, o Nordeste (Pernambuco e Bahia) participou com 46\%, São Paulo com 30\% e o Cerrado (Goiás e Minas Gerais) com 24\%. Em 2000, a área plantada reduziu-se para 14,8 mil hectares e o Cerrado transformou-se na mais importante zona de produção de tomate industrial com $77 \%$ da área plantada, seguido por São Paulo (14\%) e pelo Nordeste (9\%) (MELO e VILELA, 2004). 0 Brasil tornou-se o maior consumidor de produtos derivados de tomate da América do Sul, com importação de aproximadamente 27 mil toneladas em 2009 (SECEX, 2010).

A produção mundial de tomate para processamento industrial, em 2008, foi de aproximadamente 130 milhões de toneladas. O Brasil, um dos maiores produtores mundiais, produziu em 2008 cerca de 4 milhões de toneladas numa área de 62,12 mil hectares, indicando que a produtividade média foi de cerca de 63,34 t por hectare (FAO, 2010). A cultura do tomate no Brasil somou $20 \%$ do total de hortaliças produzidas em $7,56 \%$ da área disponibilizada e a estimativa de geração de emprego alcançou 610,25 mil no setor de produção agrícola (EMBRAPA, 2008).

O cultivo do tomateiro exige alto nível tecnológico e intensa utilização de mão-de-obra. Como os preços dos produtos derivados do tomate são muito influenciados pelo mercado internacional, a tecnologia de produção deve buscar competitividade, reduzindo custos de produção e elevando os índices de produtividade e qualidade (SILVAe GIORDANO, 2006).

O objetivo deste trabalho foi efetuar revisão de literatura sobre a contribuição do tomate na dieta alimentar, a importância da cadeia agroindustrial e seus derivados processados, a necessidade de monitoramento de agrotóxicos nos alimentos e de método simples e eficiente para análise multirresíduo.

\section{IMPORTÂNCIA DO TOMATE NA DIETA}

A busca por alimentação mais saudável e de fácil preparo tem aumentado o consumo de hortaliças no Brasil e no mundo. As hortaliças fornecem antioxidantes (vitaminas e carotenoides) que desempenham papel importante no combate aos radicais livres presentes nas células.

O consumo, per capita, de tomate no Brasil é baixo quando comparado a outros países, especialmente para tomates frescos. Segundo a Companhia de Entrepostos e Armazéns Gerais de São Paulo (CEAGESP), o consumo per capita de tomate no Brasil é de 6,5 kg por ano, enquanto na Noruega, Grécia, Suíça e outros países excede a $40 \mathrm{~kg}$ por ano (USDA, 2009).

O conhecimento da frequência da ingestão de frutas e hortaliças pela população serve para orientar estratégias locais e nacionais que estimulem o consumo desses alimentos e promovam práticas alimentares saudáveis. Em estudo sobre o consumo de frutas e hortaliças realizado no Brasil foi observada ingestão abaixo das recomendações (JAIME e MONTEIRO, 2005).

Estudos epidemiológicos mostram que o aumento do consumo de alimentos ricos em carotenoides, como o tomate, está relacionado com a diminuição do risco de várias doenças (GIOVANNUCCl et al., 2002) devido à capacidade do licopeno e outros componentes antioxidantes em prevenir danos às células mediante interações sinérgicas (FRIEDMAN, 2002). Foi demonstrado que o licopeno fornece a melhor proteção contra o oxigênio singlete, causador de danos às células e que a dieta rica em tomates e seus derivados diminue o risco de vários tipos de câncer (MARKOVIC, HRUSKAR e VAHCIC, 2006). 
Com base em estudos, a ingestão diária de 5 a $10 \mathrm{mg}$ de licopeno foi recomendada para reduzir a peroxidação lipídica e proteger as células contra danos oxidativos, bem como para redução do risco de doenças crônicas (RAO e SHEN, 2002). Essa ingestão pode ser alcançada pelo consumo diário de tomate fresco (aproximadamente $150 \mathrm{~g}$ ) ou de produtos industrializados de tomate (aproximadamente $50 \mathrm{~g}$ de purê).

\subsection{COMPOSIÇÃO NUTRICIONAL}

Existe variação na composição nutricional dos tomates devido a fatores como variedade/cultivar, estágio de maturação, clima ou estação do ano da colheita e técnicas de produção. A Tabela 1 apresenta a composição centesimal do tomate e seus derivados.

TABELA 1 - COMPOSIÇÃO EM NUTRIENTES E ENERGIA DO TOMATE (Lycopersicum esculentum mill) CRU E PROCESSADO (em $100 \mathrm{~g}$ )

\begin{tabular}{|c|c|c|c|c|c|c|}
\hline Composição & Unidade & $\begin{array}{l}\text { Tomate } \\
\text { cru }\end{array}$ & $\begin{array}{l}\text { Molho } \\
\text { industrializado } \\
\text { de Tomate }\end{array}$ & $\begin{array}{l}\text { Extrato } \\
\text { de } \\
\text { Tomate }\end{array}$ & $\begin{array}{l}\text { Purê } \\
\text { de } \\
\text { Tomate }\end{array}$ & Catchup \\
\hline Umidade & $\%$ & $95,1^{a}$ & $88,1^{a}$ & $79,7^{a}$ & $90,8^{a}$ & $63,90^{c}$ \\
\hline Energia & Kcal & $15^{a}$ & $38^{a}$ & $61^{a}$ & $28^{a}$ & $131^{c}$ \\
\hline Energia & $\mathrm{kJ}$ & $64^{a}$ & $161^{a}$ & $255^{a}$ & $117^{a}$ & $548^{c}$ \\
\hline Proteínas & $\mathrm{g}$ & $1,1^{\mathrm{a}}$ & $1,4^{\mathrm{a}}$ & $2,4^{a}$ & $1,4^{a}$ & $1,32^{c}$ \\
\hline Lipídeos & $\mathrm{g}$ & $0,2^{\mathrm{a}}$ & $0,9^{a}$ & $0,2^{a}$ & $\operatorname{Tr}^{\mathrm{a}}$ & $0,16^{c}$ \\
\hline Colesterol & $\mathrm{mg}$ & $N A^{a}$ & $N A^{a}$ & $N A^{a}$ & $N A^{a}$ & \\
\hline Carboidratos & $\mathrm{g}$ & $3,1^{\mathrm{a}}$ & $7,7^{\mathrm{a}}$ & $15,0^{a}$ & $6,9^{a}$ & $32,96^{c}$ \\
\hline Fibra & g & $1,2^{\mathrm{a}}$ & $3,1^{\mathrm{a}}$ & $2,8^{a}$ & $1,0^{\mathrm{a}}$ & $1,8^{\mathrm{C}}$ \\
\hline $\begin{array}{l}\text { Alimentar } \\
\text { Cinzas }\end{array}$ & q & $05^{a}$ & & & & $166^{c}$ \\
\hline Cálcio & $\begin{array}{l}\mathrm{g} \\
\mathrm{mg}\end{array}$ & $7^{a}$ & $\begin{array}{l}1,9^{a} \\
12^{a}\end{array}$ & $\begin{array}{l}2,0^{a} \\
29^{a}\end{array}$ & $\begin{array}{l}1,0^{a} \\
13^{a}\end{array}$ & 1,66 \\
\hline$\beta$-caroteno & $\mathrm{mg}$ & $0,32^{b}$ & $0,64^{b}$ & $0,66^{b}$ & $0,44^{b}$ & $0,35^{\mathrm{b}}$ \\
\hline Licopeno & $\mathrm{mg}$ & $3,5^{\mathrm{b}}$ & $11,2^{b}$ & $16,4^{b}$ & $11,4^{b}$ & $10,3^{b}$ \\
\hline
\end{tabular}

NA = não aplicável; $\mathrm{Tr}=$ traço.

a NEPA/UNICAMP, 2006.

${ }^{b}$ RODRIGUEZ-AMAYA et al., 2008.

'USP/FCF, 2008.

Os carotenoides são os compostos responsáveis pela cor amarela ou laranja de vários frutos e vegetais. O extensivo sistema de duplas ligações conjugadas proporciona aos carotenoides algumas de suas funções biológicas, como a atividade antioxidante. Essa estrutura altamente insaturada torna tais pigmentos sensíveis ao oxigênio, à luz e ao calor (STAHL e SIES, 1999).

O licopeno constitui o carotenoide predominante em tomates vermelhos, mas â-caroteno, $\alpha$-caroteno e luteína representam cerca de $20 \%$ dos carotenoides totais em tecido de tomate fresco (DAVIS, FISH e PERKINS-VEAZIE, 2003). O licopeno é visto como o carotenoide com maior capacidade sequestrante do oxigênio singlete, possivelmente devido à presença das duas ligações duplas não conjugadas, o que the oferece maior reatividade (STAHL e SIES, 1999).

Os antioxidantes atuam diferentemente na proteção dos organismos, podendo impedir a formação de radicais livres, interceptar os radicais livres gerados pelo metabolismo celular ou por 
fontes exógenas, evitando a formação de lesões e perda da integridade celular. Também podem reparar lesões causadas pelos radicais livres, removendo danos da molécula de DNA e reconstituindo membranas celulares danificadas. O tomate contém antioxidantes, tais como ácido ascórbico, â-caroteno, ácido clorogênico, rutina, plastoquinonas, tocoferol e xantofila, bem como elementos traços de cobre, ferro e cromo que podem atuar sinergicamente com o licopeno na prevenção de danos às células (FRIEDMAN, 2002).

\section{INDUSTRIALIZAÇÃO}

A cadeia produtiva do tomate privilegia as relações entre agropecuária, indústria de transformação e distribuição do produto. Envolve, desde a aquisição de insumos, a produção da matéria-prima, o processamento primário e secundário e a distribuição do produto final, conforme esquematizado no Fluxograma 1.

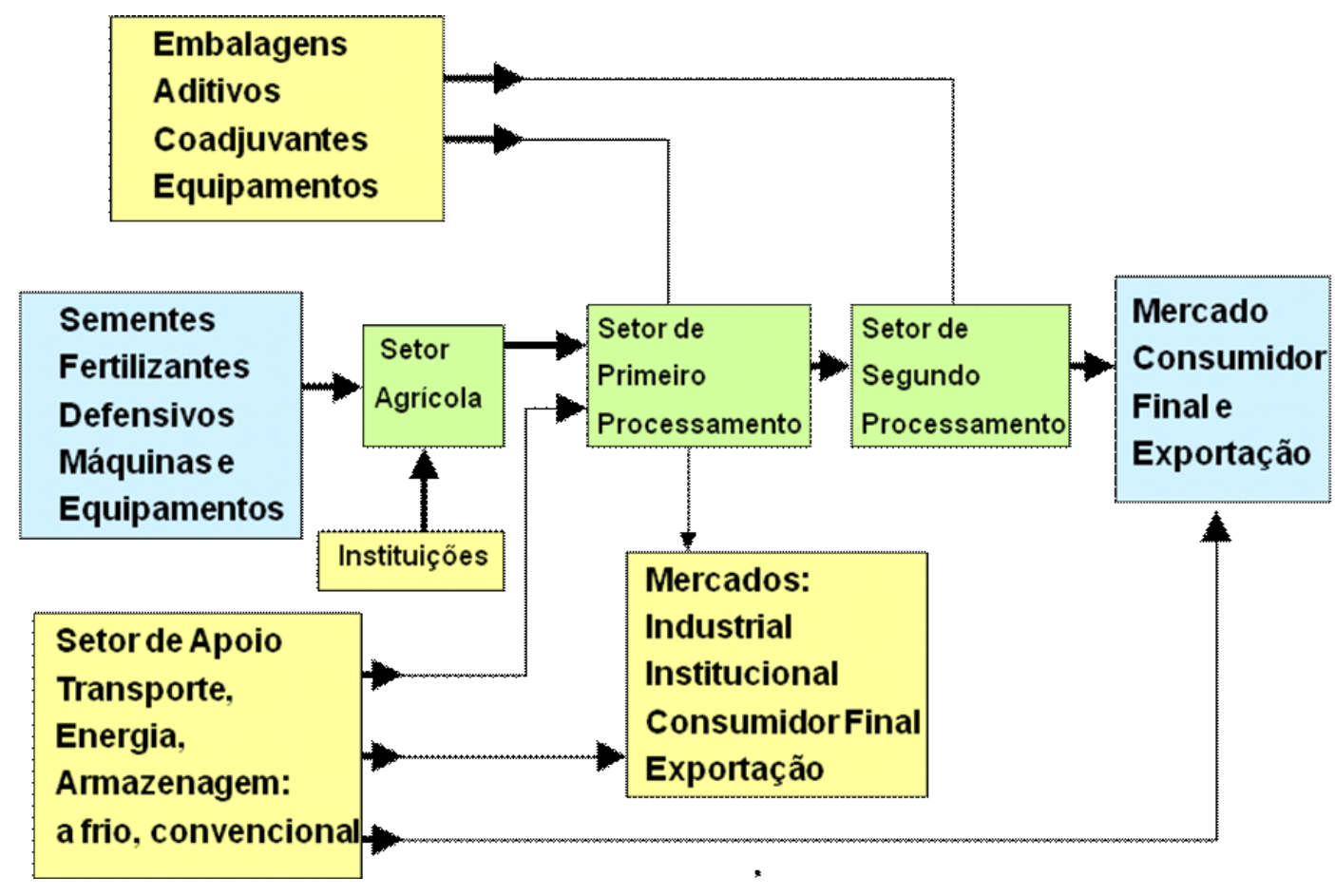

\section{FLUXOGRAMA 1 - CADEIA AGROINDUSTRIAL DO TOMATE}

FONTE: NUEVO (1994).

O processamento adequado do tomate gera diversos produtos, alguns com elevado consumo no Brasil. Pode-se obter tomate despelado, tomate seco, suco, purê, polpa concentrada, extrato, "catchup", molhos culinários diversos, e inclusive tomate em pó. A produção mundial de tomate processado em 2009 foi de aproximadamente 42 milhões de toneladas, estimando-se 39 milhões de toneladas para 2010 (WPTC, 2010).

Concentrado de tomate, produto obtido da polpa de frutos do tomateiro (Lycopersicum esculentum L.), deve conter no mínimo $6 \%$ de sólidos solúveis naturais de tomate, podendo ser adicionado de sal e/ou açúcar. O produto "catchup" deve ser elaborado a partir da polpa de frutos maduros do tomateiro, podendo ser adicionados outros ingredientes desde que não descaracterizem 
o produto. Já o extrato de tomate resulta da concentração da polpa de frutos maduros do tomateiro Solanum lycoperaicum por processo tecnológico adequado (também denominado "massa de tomate" ou "concentrado de tomate"), sendo tolerada a adição de $1 \%$ de açúcar e de $5 \%$ de cloreto de sódio. O extrato de tomate, de acordo com a sua concentração, é classificado em purê de tomate (mínimo $9 \% \mathrm{p} / \mathrm{p}$ ), extrato de tomate simples concentrado (mínimo $18 \% \mathrm{p} / \mathrm{p}$ ), extrato de tomate duplo concentrado (mínimo 25\% p/p) e extrato de tomate triplo concentrado (mínimo 35\% p/p) (ANVISA, 2003).

O processamento térmico serve para inativar micro-organismos e enzimas e estender a vida útil dos produtos (HSU, 2008). O tratamento térmico durante o processamento pode causar mudanças nas características organolépticas e nutricionais do tomate e seus derivados, devido à co-oxidação dos carotenoides e reações de Maillard (SERVILI et al., 2000).

A biodisponibilidade do licopeno aumenta durante o processamento térmico, pois ocorre a quebra das paredes celulares permitindo a extração do licopeno dos cromoplastos (MARKOVIC, HRUSKAR e VAHCIC, 2006). Essa quebra enfraquece as forças de ligação entre o licopeno e o tecido da matriz, tornando o licopeno mais acessível e reforçando a isomerização cis (SHI e LE MAGUER, 2000). Outros antioxidantes presentes no tomate, como ácido ascórbico, tocoferol e â-caroteno são mais sensíveis à degradação térmica (ABUSHITA, DAOOD e BIACS, 2000). O licopeno pode sofrer degradação durante o processamento, alterando a cor e a qualidade sensorial dos produtos à base de tomate, sendo importante investigar a estabilidade e biodisponibilidade do licopeno no tomate e seus derivados para não afetar os benefícios à saúde propiciados por esse componente aos consumidores (SHI e LE MAGUER, 2000).

Ponto crítico na cadeia agroindustrial do tomate envolve a perda pós-colheita. As causas das diferentes variações de perdas durante $o$ ano podem ser naturais e ou provocadas. As causas naturais são atribuídas aos fatores climáticos, que podem acelerar a senescência dos produtos e favorecer o desenvolvimento de patógenos causadores de apodrecimento. As causas provocadas podem ser atribuídas às embalagens inadequadas e ao manuseio incorreto do produto. No Brasil, estudos demonstram que os níveis médios de perdas pós-colheita são de $35 \%$, chegando até a $40 \%$, enquanto em outros países como os Estados Unidos da América não ultrapassam 10\% (VILELA et al., 2003).

O tempo entre a colheita e o consumo do tomate estende-se e por várias semanas, e reações fitoquímicas podem alterar o nível de atividades biológicas e medicinais de compostos como o licopeno. Portanto, as condições ambientais da pós-colheita devem ser cuidadosamente consideradas para a avaliação de compostos bioativos (JAVANMARDI e KUBOTA, 2006).

O uso doméstico de produtos à base de tomate aumentou durante os anos 90 , mas estabilizouse no decorrer da década. O consumo per capita aumentou de $29 \mathrm{~kg}$ nos anos 80 para $34 \mathrm{~kg} \mathrm{na}$ década seguinte. Esse aumento pode ser atribuído à contínua expansão da demanda de comida comprada em restaurantes e "fast food", em especial pizzas e tacos, além da conscientização da população dos benefícios à saúde de dieta rica em produtos à base de tomate (LUCIER et al., 2000).

Embora o Brasil esteja entre os maiores produtores mundiais de tomate, sua inserção no mercado internacional é baixa, tanto no segmento de produto fresco quanto no de pasta de tomate. Tal fato justifica-se pelo alto custo do produto nacional, grande distância dos países consumidores e barreiras comerciais (CARVALHO e PAGLIUCA, 2007).

\section{RESÍDUOS DE AGROTÓXICOS}

O emprego de agrotóxicos na agricultura gera benefícios econômicos, uma vez que assegura o controle e combate de pragas e doenças, protegendo a qualidade da produção. Por outro lado, os consumidores desses produtos agrícolas podem estar expostos aos agrotóxicos, devido sua presença nos alimentos. Entre os efeitos nocivos causados ao homem por essas substâncias podem ser citados: danos ao sistema nervoso central, problemas no sistema reprodutivo e locomotor, e deficiência mental, entre outros (ALDER et al., 2006). 
O estabelecimento e a regulamentação de limites máximos de resíduos (LMR) em alimentos por agências governamentais de diversos países e pela Comissão da União Européia têm se tornado mais frequente na última década. Esses procedimentos, em geral, visam garantir a segurança dos alimentos e a regulamentação do comércio externo. A Agência Nacional de Vigilância Sanitária (ANVISA), do Ministério da Saúde, é o órgão responsável pelo estabelecimento do LMR para as culturas comercializadas no Brasil (ANVISA, 2010).

O monitoramento de agrotóxicos nos alimentos constitui ferramenta importante para a caracterização e o gerenciamento dos riscos decorrentes do uso desses produtos nas condições reais de aplicação. O monitoramento do uso de agrotóxicos no campo não é tarefa fácil, podendo ocorrer uso inadequado de ingredientes ativos nas culturas, ou colheita antes do término do período de carência do produto aplicado (GODOY e OLIVEIRA, 2004).

A ANVISA iniciou em 2001, o Programa de Análise de Resíduos de Agrotóxicos em Alimentos (PARA). Os resultados observados nas culturas monitoradas pelo PARA dividem-se em duas categorias: resíduos que podem causar dano à saúde porque excederam os limites máximos estabelecidos pela legislação e os resíduos de produtos não autorizados para aquela cultura. $O$ percentual de resultados insatisfatórios para o tomate em 2006 foi de 2,01\%, alcançou 44,72\% em 2007, baixou para 18,27\% em 2008 e elevou-se para 32,6\% em 2009. A continuidade do PARAé importante para garantir a segurança dos alimentos consumidos pela população, identificar as fontes de contaminação, proporcionar avaliação quanto ao uso inadequado e não autorizado de agrotóxicos, estimular a adoção de Boas Práticas Agrícolas, bem como fornecer subsídios para possível reavaliação dos agrotóxicos (ANVISA, 2010).

\subsection{MÉTODOS DE DETECÇÃO DE RESÍDUOS DE AGROTÓXICOS}

Existem muitos métodos de análises multirresíduos de agrotóxicos para produtos agrícolas, mas é fundamental que a técnica possa extrair dezenas ou mesmo centenas de substâncias de matrizes complexas, que grande quantidade de interferentes co-extraídos possam ser eliminados na etapa do "clean-up", e que as técnicas analíticas de detecção adotadas sejam indicadas para a quantificação dos analitos (PANG et al., 2006).

A análise de resíduos de agrotóxicos, além de representar instrumento de proteção à saúde humana, está relacionada a fatores econômicos. Estima-se que no mundo, cerca de 200.000 amostras de alimentos sejam analisadas anualmente para resíduos de agrotóxicos com diversos propósitos (LEHOTAY, 2005).

No passado, métodos baseados em cromatografia a líquido $(C L)$ eram pouco aplicados para análise de resíduos porque os detectores tradicionais ultravioleta (UV), arranjo de diodo e fluorescência são menos seletivos e sensíveis do que instrumentos de cromatografia a gás (CG). A disponibilidade da ionização de pressão atmosférica (atmospheric pressure ionization - API) causou espetacular mudança, pois em combinação com a espectrometria de massas (EM) aumentou a sensibilidade da detecção $C L$ em várias ordens de grandeza, comparado aos tradicionais detectores eletrospray (electrospray ionization - ESI) e ionização química a pressão atmosférica (atmospheric pressure chemical ionization - APCI). Além disso, as técnicas de troca de coluna da CL e extensos processos de limpeza da amostra tornam-se desnecessários se o acoplamento EM-EM for utilizado e operado no modo de monitoração de reação selecionada (selected reaction monitoring - SRM) (ALDER et al., 2006).

A análise de resíduos de agrotóxicos em alimentos é dificultada por diversos fatores, tais como demora, necessidade de grandes volumes de solvente orgânico, várias etapas de extração, tornando-a extremamente laboriosa (FRENICH, BOLAÑOS e VIDAL, 2007).

Dentre as técnicas de extração normalmente utilizadas para análises de resíduos destacamse a extração líquido-líquido (liquid-liquid extraction - LLE), a extração com fluido supercrítico (supercritical fluid extraction - SFE), a extração em fase sólida (solid phase extraction - SPE), a dispersão da matriz em fase sólida (matrix solid phase dispersion - MSPD), a micro extração em fase sólida (solid phase micro-extraction - SPME) e a extração em fase sólida dispersiva (dispersive solid-phase extraction dSPE) (BARRIONUEVO e LANÇAS, 2001; ANASTASSIADES e SCHERBAUM, 2005). 
Vários estudos foram realizados para comparar os diferentes métodos de extração para análise de alimentos e apontar metodologias mais fáceis. Díez et al. (2006) compararam os métodos de QuEChERS, empregando acetonitrila na extração, o método mini Luke; o método de extração com acetato de etila, que envolve posterior clean-up por cromatografia de permeação em gel (gel permeation chromatography - GPC) e, ainda, a simples extração em acetona para verificar as diferenças entre os métodos na análise de cevada. Os resultados apresentados pelo método QuEChERS, com menor tempo de extração e pequeno volume de solvente orgânico, mostraram-se muito promissores em comparação a extrações por procedimentos mais complicados (DÍEZ et al., 2006).

Desde 2003, o método QuEChERS (iniciais das palavras Quick, Easy, Cheap, Effective, Rugged, Safe) vem sendo empregado para análise de pesticidas em diferentes matrizes de alimentos como: banana (HERNÁNDEZ-BORGES et al., 2009), uva, mosto e vinho (CUNHA et al., 2009), ovos, leite e abacate (LEHOTAY, MASTOVSKA e YUN, 2005), incluindo o tomate (LIU et al., 2005; KMELLAR et al., 2008; SCHENCK et al., 2009) com boa sensibilidade e seletividade. Esse método foi oficializado em 2007 pela Association of Official Analytical Chemists (AOAC) para a determinação de resíduos de pesticidas em alimentos (AOAC, 2007), sendo também oficializado pelo European Committee for Standardization (EUROPEAN..., 2008). No Brasil, esse método foi selecionado para ser implementado no Laboratório de Resíduos e Contaminantes (LRC) da Embrapa Meio Ambiente e no Instituto de Tecnologia de Pernambuco (ITEP) para monitoramento e avaliação de resíduos de agrotóxicos, visando atender ao mercado exportador de frutas. Tais fatos evidenciam o potencial do método para aplicação em análises de rotina.

\section{CONSIDERAÇÕES FINAIS}

O cultivo do tomate no Brasil é muito expressivo, principalmente, na região centro-oeste e sua cadeia produtiva está entre as mais importantes no âmbito do agronegócio brasileiro. Embora seja um dos maiores produtores de tomate, o Brasil tem baixa inserção no mercado internacional devido ao alto custo do produto nacional e barreiras comerciais.

O tomate e seus derivados são a principal fonte de licopeno da dieta dos brasileiros e tem sido observada ingestão abaixo das recomendações. É importante orientar estratégias que estimulem o consumo desses alimentos e promovam práticas alimentares saudáveis, além da conscientização da população sobre os benefícios à saúde da dieta rica em produtos à base de tomate.

É possível obter, mediante processamento, várias opções para consumo do tomate. O processamento é útil inclusive para evitar as perdas pós-colheita e em toda a cadeia agroindustrial, que podem alcançar até $40 \%$ do total da produção. Para aumentar a disponibilidade de alimentos para toda a população é necessária a participação da sociedade e de produtores em programas educativos, além da transferência e adoção de tecnologias para a redução de perdas e desperdícios.

O processamento térmico aumenta a biodisponibilidade do licopeno, que é extraído dos cromoplastos, e produtos industrializados de tomate apresentam maiores quantidades de licopeno.

Considerando os dados do programa de monitoramento, a aplicação de agrotóxicos não autorizados e em reavaliação nas culturas vem sendo realizada de maneira indiscriminada. Essa prática expõe o trabalhador rural a agrotóxicos com elevada toxicidade aguda e/ou crônica e aumenta o risco dietético de consumo de resíduos desses produtos, já que não são computados no cálculo do impacto na Ingestão Diária Aceitável. É importante a rastreabilidade desses produtores para permitir a responsabilização nos diferentes elos da cadeia produtiva.

Deve-se salientar a importância de serem desenvolvidos métodos de detecção de agrotóxicos rápidos e econômicos para o cumprimento das medidas sanitárias e fitossanitárias exigidas. $\mathrm{A}$ disponibilização de métodos multirresíduos que promovam a detecção de várias classes de agrotóxicos em matrizes complexas, como o QuEChERS, oferecem várias vantagens na determinação de resíduos de agrotóxicos em alimentos, podendo ser usado como método de rotina em diversos laboratórios. 


\section{ABSTRACT \\ THE TOMATO AS FOOD - AGROINDUSTRIAL CHAIN AND PESTICIDE RESIDUES}

The aim of this work was to perform a literature review about the importance of tomato and its derivatives in the diet, present the influence of the tomato agroindustrial chain on the agrobussines sphere in Brazil, and indicate an efficient method for detection of pesticide residues in this matrix. For its safe consumption are needed, in addition to the application of good agricultural practices in their cultivation, information on the monitoring of pesticide residues to ensure the quality of tomatoes subjected to treatment with pesticides. The availability of multiresidue methods, such as QuEChERS, that promote the detection of different categories of pesticides in complex matrixes offers several advantages on determination of pesticides residue in food allowing its use as a routin method on several laboratories.

KEY-WORDS: TOMATOES; LYCOPENE; PESTICIDE RESIDUES; MULTIRESIDUE ANALYSIS.

\section{REFERÊNCIAS}

1 ABUSHITA, A.A; DAOOD, H.G.; BIACS, P.A. Change in carotenoids and antioxidant vitamins in tomato as a function of varietal and technological factors. Journal of Agricultural and Food Chemistry, v.48, n.6, p.2075-2081, 2000.

2 ALDER, L.; GREULICH, K.; KEMPE, G.; VIETH, B. Residue analysis of 500 high priority pesticides: better by GC-MS or LC-MS/MS? Mass Spectrometry Reviews, v.25, n.6, p.838-865, 2006.

3 ANASTASSIADES, M.; SCHERBAUM, E. Sample handling and clean-up procedures. II New developments. In: FERNANDEZ-ALBA, A.R. (Ed.). Chromatographic-mass spectrometric food analysis for trace determination of pesticide residues. Amsterdam: Elsevier, 2005. cap.4, p.113-233. (Comprehensive Analytical Chemistry, 43).

4 ANVISA. Agência Nacional de Vigilância Sanitária. Alimentos - legislação específica de alimentos - regulamentos técnicos por assuntos. 2003. Disponível em:<http://www.anvisa.gov.br/alimentos/legis/especifica/regutec. htm2003>. Acesso em: 24/05/10.

5 ANVISA. Agência Nacional de Vigilância Sanitária. Programa de monitoramento de resíduos de agrotóxicos em alimentos - PARA. 2010. Disponível em: <http://www.anvisa.gov.br/reblas/para/index.htm2010>. Acesso em: 30/06/10.

6 AOAC. Association of Official Analytical Chemists. Methods Committee Reports. Official method 2007.01: pesticide residues in foods by acetonitrile extraction and partitioning with magnesium sulphate. Disponível em: <http:// www.aoac.org/oma/rev_2_pdfs /2007_01.pdf>. Acesso em: 30/06/10.

7 BARRIONUEVO, W.R.; LANÇAS, F.M. Extração em fase sólida (SPE) e micro extração em fase sólida (SPME) de piretróides em água. Química Nova, v.24, p.172-175, 2001.

8 CARVALHO, J.L.; PAGLIUCA, L.G. Tomate: um mercado que não pára de crescer globalmente. Hortifruti Brasil, Piracicaba, v.6, n.58, p.6-14, 2007.

9 CASQUET, E. Princípios de economia agrária. Zaragoza: Acribia, 1998. p.368.

10 CUNHA, S.C.; FERNANDES, J.O.; ALVES, A.; OLIVEIRA, M.B.P.P. Fast low-pressure gas chromatography-mass spectrometry method for the determination of multiple pesticides in grapes, musts and wines. Journal of Chromatography A, Amsterdam, v.1216, n.1, p.119-126, 2009

11 DAVIS, A.R.; FISH, W.W.; PERKINS-VEAZIE, P. A rapid spectrophotometric method for analyzing lycopene content in tomato and tomato products. Postharvest Biology and Technology, v.28, n.3, p.425-430, 2003.

12 DÍEZ, C.; TRAAG, W.A.; ZOMMER, P.; MARINERO, P.; ATIENZA, J. Comparison of an acetonitrile extraction/partitioning and "dispersive solid-phase extraction" method with classical multi-residue methods for the extraction of herbicide residues in barley samples. Journal of Chromatography A, Amsterdam, v.1131, n.1-2, p.11-23, 2006.

13 EMBRAPA. Empresa Brasileira de Pesquisa Agropecuária. Situação da produção de hortaliças no Brasil - 2008. Disponível em:<http://www.cnph.embrapa.br/paginas/hortalicas_em_numeros/hortalicas_em_numeros.htm2010>. Acesso em: 21/05/10.

14 EUROPEAN COMMITTEE FOR STANDARDIZATION. CEN/TC 275 15662:2008: foods of plant origin - determination of pesticide residues using GC-MS and/or LC-MS/MS following acetonitrile extraction/partitioning and clean-up by dispersive SPE - QuEChERS-method. Disponível em:<https://www.astandis.at/shopV5/Preview.action \%3Bjsessionid =0591FE94A94C9C6E7117064F9A22C0CE? preview=\&dokkey=321612\&selectedLocale=en>. Acesso em: 30/06/10. 
15 FAO. Food and Agriculture Organization. Production. Disponível em:<http://www.faostat.fao.org/site/567/ DesktopDefault.aspx?PagelD=567\#ancor2010>. Acesso em: 14/05/10.

16 FRENICH, A.G.; BOLAÑOS, P.P.; VIDAL, J.L.M. Multiresidue analysis of pesticides in animal liver by gas chromatography using triple quadruple tandem mass spectrometry. Journal of Chromatography A, Amsterdam, v.1153, n.1-2, p.194-202, 2007.

17 FRIEDMAN, M. Tomato glycoalkaloids: role in the plant and in the diet. Journal of Agricultural and Food Chemistry, v.50, n.21, p.5751-5780, 2002.

18 GIOVANNUCCI, E.; RIMM, E.B.; LIU, Y.; STAMPFER, M.J.; WILLETT, W.C. A prospective study of tomato products, lycopene, and prostate cancer risk. Journal of the National Cancer Institute, v.94, n.5, p.391-398, 2002.

19 GODOY, R.C.B.; OLIVEIRA, M.I. Agrotóxicos no Brasil: processo de registro, riscos à saúde e programas de monitoramento. Cruz das Almas: Embrapa Mandioca e Fruticultura Tropical, 2004. (Documentos, 134). Disponível em: <http://www.cnpmf.embrapa.br/publicacoes/documentos/documento_134.pdf>. Acesso em: 17/05/10

20 HERNÁNDEZ-BORGES, J.; CABRERA, J.C.; RODRÍGUEZ-DELGADO, M.A.; HERNÁNDEZ-SUÁREZ, E.M.; SAÚCO, V.G. Analysis of pesticide residues in bananas harvested in the Canary Islands (Spain). Food Chemistry, Barking, v.113, n.1, p.313-319, 2009.

21 HSU, K.C. Evaluation of processing qualities of tomato juice induced by thermal and pressure processing. Food Science and Technology, v.41, n.3, p.450-459, 2008.

22 JAVANMARDI, J.; KUBOTA, C. Variation of lycopene, antioxidant activity, total soluble solids and weight loss of tomato during postharvest storage. Postharvest Biology and Technology, v.41, n.2, p.151-155, 2006.

23 JAIME, P.C.; MONTEIRO, C.A. Fruit and vegetable intake by Brazilian adults, 2003. Cadernos de Saúde Pública, v.21, p.19-24, 2005.

24 KMELLAR, B.; FODOR, P.; PAREJA, L.; FERRER, C.; MARTINEZ-UROZ, M.A.; VALVERDE, A.; FERNANDEZ-ALBA, A.R. Validation and uncertainty study of a comprehensive list of 160 pesticide residues in multi-class vegetables by liquid chromatography-tandem mass spectrometry. Journal of Chromatography A, Amsterdam, v.1215, n.1-2, p.37-50, 2008.

25 LEHOTAY, S.J.; Quick, easy, cheap, effective, rugged, and safe approach for determining pesticide residues. In: MARTÍNEZ VIDAL, J.L.; FRENICH, A.G. (Ed.). Pesticide protocols. Totowa: Humana Press, 2005. p.239-261. (Methods in Biotechnology, 19).

26 LEHOTAY, S.J.; MASTOVSKA, K.; YUN, S.J. Evaluation of two fast and easy methods for pesticide residue analysis in fatty food matrixes. Journal of AOAC International, Arlington, v.88, n.2, p.630-638, 2005.

27 LIU, M.; HASHI, Y.; SONG, Y.; LIN, J.M. Simultaneous determination of carbamate and organophosphorus pesticides in fruits and vegetables by liquid chromatography-mass spectrometry. Journal of Chromatography A, Amsterdam, v.1097, n.1-2, p.183-187, 2005.

28 LUCIER, G.; LIN, B.H.; ALLSHOUSE, J.; KANTOR, L.S. Factors affecting tomato consumption in the United States. Economic Research Service/ USDA, 2000. Disponível em: < http://www.ers.usda.gov/briefing /vegetables/vegpdf/ TomatoConsumption.pdf>. Acesso em: 30/06/10.

29 MARKOVIC, K.; HRUSKAR, M.; VAHCIC, N. Lycopene content of tomato products and their contribution to the lycopene intake of Croatians. Nutrition Research, v.26, n.11, p.556-560, 2006.

30 MELO, P.C.T.; VILELA, N.J. Desempenho da cadeia agroindustrial brasileira do tomate na década de 90 . Horticultura Brasileira, v.22, p.154-160, 2004.

31 NEPA/UNICAMP. Núcleo de Estudos e Pesquisas em Alimentação/ Universidade Estadual de Campinas. Tabela brasileira de composição de alimentos - TACO Versão 2. p.28-29. Disponível em:<http://www.unicamp.br/ nepa/taco/tabela.php?ativo=tabela2006>. Acesso em: 15/05/10.

32 NUEVO, P. A. S. Aspectos da cadeia agroindustrial do tomate no Brasil. Informações Econômicas, v.24. n.2, p.3144, 1994.

33 PANG, G.F.; CAO, Y.Z.; ZHANG, J.J.; FAN C.L.; LIU, Y.M.; LI, X.M.; JIA, G.Q.; LI, Z.Y.; SHI, Y.Q.; WU, Y.P.; GUO, T.T. Validation study on 660 pesticide residues in animal tissues by gel permeation chromatography clean up/gas chromatography-mass spectrometry and liquid chromatography-tandem mass spectrometry. Journal of Chromatography A, Amsterdam, v.1125, p.1-30, 2006.

34 RAO, A.V.; SHEN, H. Effect of low dose lycopene intake on lycopene bioavailability and oxidative stress. Nutrition Research, v.22, n.10, p.1125-1131, 2002.

35 RODRIGUEZ-AMAYA, D.B.; KIMURA, M.; GODOY, H.T.; AMAYA-FARFAN, J. Updated Brazilian database on food carotenoids: factors affecting carotenoid composition. Journal of Food Composition and Analysis, v.21, n.6, p.445-463, 2008. 
36 SCHENCK, F.; WONG, J.; LU, C.S.; LI, J.; HOLCOMB, J.R.; MITCHELL, L.M. Multiresidue analysis of 102 organophosphorus pesticides in produce at parts-per-billion levels using a modified QuEChERS method and gas chromatography with pulsed flame photometric detection. Journal of AOAC International, Arlington, v.92, n.2, p.561-573, 2009.

37 SECEX. Secretaria de Comércio Exterior. Sistema de análise das informações de comércio exterior via internet. Disponível em: <http://aliceweb.desenvolvimento.gov.br/alice.asp2010>. Acesso em: 26/05/10.

38 SERVILI, M.; SELVAGGINI, R.; TATICCHI, A.; BEGLIOMINI, A.L.; MONTEDORO, G. Relationships between the volatile compounds evaluated by solid phase microextraction and the thermal treatment of tomato juice: optimization of the blanching parameters. Food Chemistry, Barking, v.71, n.3, p.407-415, 2000.

$39 \mathrm{SHI}$, J.; LE MAGUER, M. Lycopene in tomatoes: chemical and physical properties affected by food processing. Critical Reviews in Food Science and Nutrition, v.40, n.1, p.1-42, 2000.

40 SILVA, J.B.C.; GIORDANO, L.B. Cultivo de tomate para industrialização. EMBRAPA HORTALIÇAS, 2006. Disponível em: < http://www.cnph.embrapa.br/sistprod/tomate_/index.htm>. Acesso em: 26/05/10.

41 STAHL, W.; SIES, H. Carotenoids: occurrence, biochemical activities, and bioavailability. In: LESTER, P. et al (Ed.). Antioxidant food supplements in human health. San Diego: Academic Press, 1999. p.183-202.

42 USP/FCF. Universidade de São Paulo. Faculdade de Ciências Farmacêuticas. Departamento de Alimentos e Nutrição Experimental. Tabela brasileira de composição de alimentos. Projeto integrado de composição de alimentos. 2008. Disponível em: <http://www.fcf.usp.br/tabela/lista.asp?base=c2008>. Acesso em: 20/05/10.

43 USDA. United States Department of Agriculture. Brazil tomatoes and products annual 2009. Disponível em: < http://gain.fas.usda.gov/Recent\%20GAIN\%20Publications/Commodity\%20Report_TOMATOES \%20AND\%20PRODUCTS\%20ANNUAL_Brasilia_Brazil_5-27-2009.pdf>. Acesso em: 27/05/10.

44 VILELA, N.J.; LANA, M.M.; NASCIMENTO, E.F.; MAKISHIMA N. O peso da perda de alimentos para a sociedade: o caso das hortaliças. Horticultura Brasileira, v.21, n.2, p.141-143, 2003.

45 WPTC. World Processing Tomato Council. World processing tomato. Disponível em:<http://www.wptc.to/releases/ releases27.pdf2010>. Acesso em: 26/05/10.

\section{AGRADECIMENTOS}

Os autores agradecem à Fundação de Amparo a Pesquisa do Estado de São Paulo (FAPESP) e ao Conselho Nacional de Desenvolvimento Científico e Tecnológico (CNPq) pelo apoio financeiro. 